\title{
Subclinical infection associated with delayed union after transtrochanteric rotational osteotomy
}

\author{
This article was published in the following Dove Press journal: \\ Orthopedic Research and Reviews \\ 9 February 2012 \\ Number of times this article has been viewed
}

\section{Goro Motomura' \\ Takuaki Yamamoto' \\ Sanshiro Inoue ${ }^{2}$ \\ Yasuharu Nakashima' \\ Taro Mawatari' \\ Yukihide Iwamoto' \\ 'Department of Orthopaedic Surgery, Graduate School of Medical Sciences, Kyushu University, Fukuoka, Japan; ${ }^{2}$ Department of Orthopaedic Surgery, Miyazaki Prefectural Miyazaki Hospital, Miyazaki, Japan}

Correspondence: Takuaki Yamamoto Department of Orthopaedic Surgery, Graduate School of Medical Sciences, Kyushu University, 3-I-I Maidashi, Higashi-ku, Fukuoka 8I 28582, Japan Tel +8I92 6425488

$\mathrm{Fax}+81926425507$

Email yamataku@ortho.med.kyushu-u.ac.jp

\begin{abstract}
Infected nonunion of the transtrochanteric osteotomy site is an uncommon complication of transtrochanteric anterior rotational osteotomy. A 45-year-old male underwent anterior rotational osteotomy for osteonecrosis of the left femoral head. A radiograph obtained 4 months after surgery showed the presence of osteolytic lesions around the trochanteric wire, and the transtrochanteric osteotomy site appeared to be fuzzy. In spite of the continuation of partial weight bearing, osteolytic changes progressed. An open biopsy was performed 13 months after surgery. Samples were taken from multiple sites, including osteolytic lesions, one of which was confirmed to contain coagulase-negative staphylococcus. Nineteen days after performing thorough debridement and irrigation, the patient underwent a refixation of the osteotomy site using an iliac bone graft and a sliding hip screw system. A radiograph obtained at the final follow-up revealed bone union at the site of osteotomy.
\end{abstract}

Keywords: osteonecrosis, infected nonunion, osteotomy

\section{Introduction}

Transtrochanteric anterior rotational osteotomy (ARO) is one of the joint-preserving surgeries that can be performed for osteonecrosis of the femoral head. ${ }^{1}$ Previous reports have demonstrated the effectiveness of this method when performed with the correct indications and with accurate surgical techniques, ${ }^{2-5}$ while several postoperative complications have also been reported..$^{5,6}$

Nonunion of the transtrochanteric osteotomy site is one of the possible complications of ARO. To our knowledge, a few studies have described the incidence of nonunion cases after ARO. ${ }^{6,7}$ Sugano et $\mathrm{al}^{6}$ reported that one of 41 hips showed nonunion, which was treated by refixation using a hip-plate system. Rijnen et $\mathrm{al}^{7}$ reported that one of 26 hips was converted to total hip arthroplasty due to nonunion of the osteotomy site.

On the other hand, infected nonunion of the transtrochanteric osteotomy site is an uncommon complication of ARO, and thus, there have been no reports in the literature regarding its clinicoradiological profile. We herein describe a case of infected nonunion that was made by a positive culture of a sample taken from the osteotomy site 13 months after surgery.

\section{Case report}

A 45-year-old male presented with left hip pain during office work. He had a 17-year history of systemic lupus erythematosus, which had been treated with corticosteroids until 3 years before the onset of hip pain. He also had a history of excessive 
alcohol intake. He was eventually diagnosed with idiopathic osteonecrosis of the femoral head at a local hospital, and then was referred to our hospital for surgery.

Radiographs at his first visit demonstrated a slight degree of collapse and an obvious sclerotic rim formation in the left femoral head. T1-weighted magnetic resonance imaging showed a typical low-intensity band in the left femoral head. Since more than one-third intact posterior area of the femoral head was observed, ARO was considered to be indicated. One month after the onset of pain, the patient underwent ARO.

A cephem antibiotic was intravenously administered for prophylactic purposes for 7 days. For 5 days after surgery, an intermittent high fever (more than $38^{\circ} \mathrm{C}$ ) was observed, and by day 10 after surgery he then gradually became afebrile. The level of C-reactive protein returned to the preoperative level $(0.5-0.7 \mathrm{mg} / \mathrm{dL}$ ) within 2 weeks postoperatively (Figure 1). One-third partial weight-bearing walking was started 5 weeks after surgery as usual. Two months after surgery, the patient went back to the local hospital using two crutches. Radiographs obtained 2 months after surgery did not indicate bone union at the site of osteotomy (Figure 2A). During his stay in our hospital, the patient had not developed any symptoms that suggested deep infection, such as a relapsing high fever, delayed wound discharge, or rest pain.

After discharge from the hospital (3 months after surgery), however, the patient had experienced persistent pain around the left hip at the time of weight bearing. A radiograph obtained 4 months after surgery showed the presence of osteolytic lesions around the trochanteric wire, which had been used for reattachment of the greater trochanter (Figure 2B). In addition, the osteotomy site near the lesser trochanter appeared to be fuzzy (Figure 2B). Although his hip pain had gradually improved by 6 months postoperatively, he continued to use a crutch in accordance with our instructions.

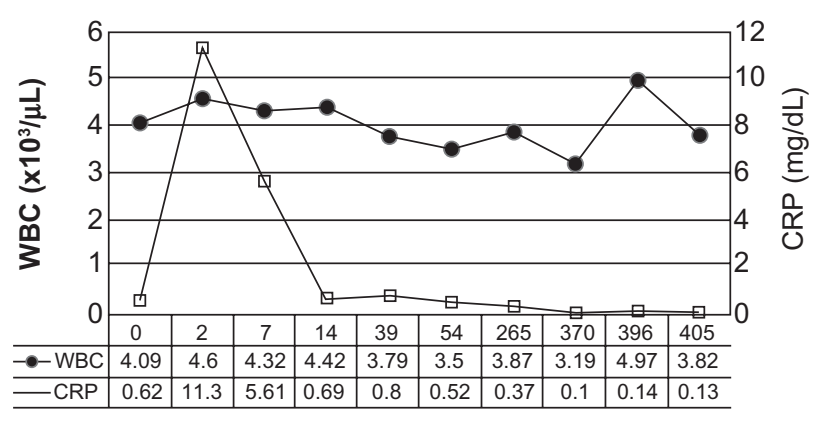

Days after the first surgery

Figure I Sequential changes in both white blood cell count (WBC) and C-reactive protein (CRP) levels.

Notes: "Day 0" means a day before the first surgery. The day of the first surgery is expressed as "Day I".

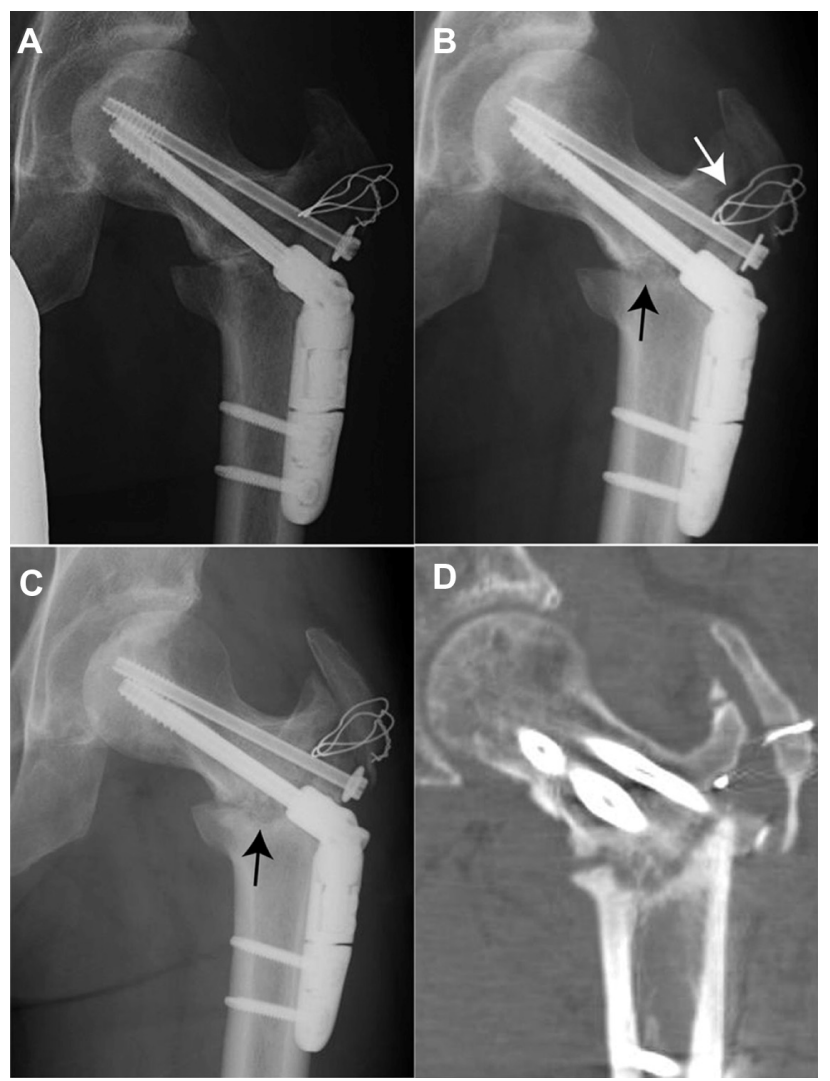

Figure 2 (A) A radiograph obtained 2 months after osteotomy had not yet indicated bone union at the site of osteotomy. (B) A radiograph obtained 4 months after osteotomy showed the presence of osteolytic lesions around the trochanteric wire (white arrow), and the osteotomy site near the lesser trochanter appeared to be fuzzy (black arrow). (C) A radiograph obtained 9 months after osteotomy revealed the progression of osteolytic changes at the site of transtrochanteric osteotomy (black arrow), associated with varus deformity with the neck-shaft angle. (D) A computed tomography scan obtained II months after osteotomy demonstrated nonunion at the site of osteotomy.

Nine months after surgery, a radiograph revealed the progression of osteolytic changes at the site of the transtrochanteric osteotomy, associated with varus deformity with the neckshaft angle (Figure 2C). A computed tomography scan obtained 11 months after surgery demonstrated nonunion at the site of transtrochanteric osteotomy (Figure 2D). The level of C-reactive protein obtained 9 months, and 1 year after surgery had been 0.37 and $0.10 \mathrm{mg} / \mathrm{dL}$, respectively (Figure 1). The erythrocyte sedimentation rate was $10 \mathrm{~mm}$ 1 year after surgery.

Thirteen months after surgery, an open biopsy was performed to examine the osteotomy site for the presence of infection. At that time, the trochanteric wire and hip screw system, both of which had been loosened, were removed. Although there was no purulent fluid, a cylindrical bone defect was seen around the drilled hole in the distal femoral fragment through which the trochanteric wire had been passed. The anterior aspect of the transtrochanteric osteotomy 
site was covered with callus, while the posterior aspect was filled with fibrous granulation tissue. Before a prophylactic antibiotic was given, samples were taken from multiple sites, including the enlarged drilled hole in the distal femoral fragment, from which coagulase-negative staphylococcus had been confirmed with enrichment culture. The isolated organism showed sensitivity to a wide range of antibiotics.

Soon after the positive culture, the patient underwent removal of the remaining screws and thorough bone debridement. Povidone-iodine solution was used to irrigate the wound with pulsatile lavage. The wound was closed in layers after the insertion of a catheter percutaneously, through which a culture-specific antibiotic was directly infused into the site of osteotomy for 4 days after the operation. Another culture-specific antibiotic was given intravenously for 8 days. Four days after discontinuation of the intravenous antibiotic, the inserted catheter was removed, and then the tip of the catheter was sent for culture. After confirmation of a negative culture, the patient underwent refixation of the osteotomy site using an iliac bone graft and a sliding hip screw system (Figure 3A). A radiograph obtained at the final follow up ( 2 years after the last surgery) revealed bone union at the site of transtrochanteric osteotomy (Figure 3B).

The patient was informed that the data from the case would be submitted for publication, and gave his consent.

\section{Discussion}

We herein present a rare case of infected nonunion at the transtrochanteric osteotomy site after ARO. Because of the minimal infectious symptoms, we initially diagnosed our case as just a delayed union, which is one of the possible complications after ARO. Since previous reports showed that

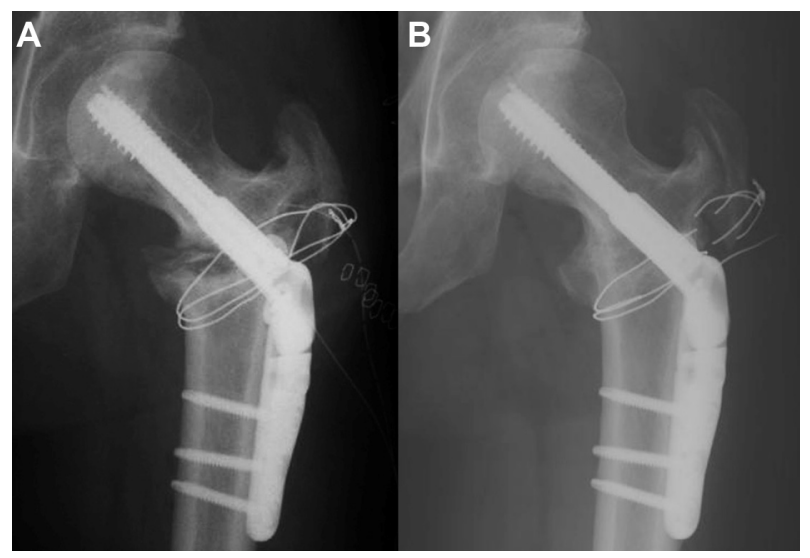

Figure 3 (A) A radiograph obtained just after the refixation of the osteotomy site using an iliac bone graft and a sliding hip screw system. (B) A radiograph obtained 2 years after the last surgery revealed bone union at the site of transtrochanteric osteotomy. delayed union after ARO normally has no need for additional surgery, ${ }^{5,8}$ we followed our case conservatively by extending the period of partial-weight bearing. However, since osteolytic changes at the site of transtrochanteric osteotomy had progressed, we performed an open biopsy because none of the examinations had completely ruled out the presence of infection. At any rate, the biopsy should have been performed at an earlier time point.

Several factors may be responsible for the subclinical manifestation in the current case. Above all, the low numbers of bacteria may be mainly associated with the condition.

We concluded that the current result of the biopsy was clinically significant based on the fact that the positivecultured sample had been taken from the enlarged drilled hole in the distal femoral fragment, in which the osteolytic changes were most evident. Also taking into consideration the fact that the organism had been isolated by enrichment culture, we speculate that both the low numbers of bacteria and the possibility of sampling error may have inevitably resulted in the remaining culture-negative specimens in our case.

Regarding the treatment of infection-related nonunion, Motsitsi ${ }^{9}$ recommended an "infection-elimination first" strategy, which involves two steps: control of infection by local radical debridement of dead tissue, followed by reconstruction. On the other hand, Rodriguez-Merchan and Forriol ${ }^{10}$ recommended concomitant treatment when possible. Although we selected the two-stage strategy, onestage treatment might also have been indicated for our case because the condition may be associated with an extremely low burden of organisms, which furthermore showed no multidrug-resistance. We believe that treatment for an infection-related nonunion after osteotomy must be tailored to the individual patient.

\section{Conclusion}

In conclusion, we would like to stress that a minor infection at the site of osteotomy should be included in the differential diagnosis of nonunion after ARO.

\section{Acknowledgments}

Our study was supported in part by a Research Grant for Intractable Diseases from the Ministry of Health and Welfare of Japan, and grant-in-aid in Scientific Research (grant 21791402, 21591948) from Japan Society for the Promotion of Science. No author or related institution has received any financial benefit from research in this study. 


\section{Disclosure}

The authors report no conflicts of interest in this work.

\section{References}

1. Sugioka Y. Transtrochanteric anterior rotational osteotomy of the femoral head in the treatment of osteonecrosis affecting the hip: a new osteotomy operation. Clin Orthop Relat Res. 1978;130:191-201.

2. Decaux G. Long-term treatment of patients with inappropriate secretion of antidiuretic hormone by the vasopressin receptor antagonist conivaptan, urea, or furosemide. Am J Med. 2001;110(7):582-584.

3. Motomura G, Yamamoto T, Suenaga K, et al. Long-term outcome of transtrochanteric anterior rotational osteotomy for osteonecrosis of the femoral head in patients with systemic lupus erythematosus. Lupus. 2010; 19(7):860-865

4. Miyanishi K, Noguchi Y, Yamamoto T, et al. Prediction of the outcome of transtrochanteric rotational osteotomy for osteonecrosis of the femoral head. J Bone Joint Surg Br. 2000;82(4):512-516.
5. Sugioka $\mathrm{Y}$, Hotokebuchi T, Tsutsui H. Transtrochanteric anterior rotational osteotomy for idiopathic and steroid-induced necrosis of the femoral head. Indications and long-term results. Clin Orthop Relat Res. 1992;(277):111-120.

6. Sugano N, Takaoka K, Ohzono K, Matsui M, Saito M, Saito S. Rotational osteotomy for non-traumatic avascular necrosis of the femoral head. J Bone Joint Surg Br. 1992;74(5):734-739.

7. Rijnen WH, Gardeniers JW, Westrek BL, Buma P, Schreurs BW. Sugioka's osteotomy for femoral-head necrosis in young Caucasians. Int Orthop. 2005;29(3):140-144.

8. Ha YC, Kim HJ, Kim SY, Kim KC, Lee YK, Koo KH. Effects of age and body mass index on the results of transtrochanteric rotational osteotomy for femoral head osteonecrosis. J Bone Joint Surg Am. 2010; 92(2):314-321.

9. Motsitsi NS. Management of infected nonunion of long bones: the last decade (1996-2006). Injury. 2008;39(2):155-160.

10. Rodriguez-Merchan EC, Forriol F. Nonunion: general principles and experimental data. Clin Orthop Relat Res. 2004;419:4-12.

\section{Publish your work in this journal}

Orthopedic Research and Reviews is an international, peer-reviewed, open access journal focusing on the patho-physiology of the musculoskeletal system, trauma, surgery and other corrective interventions to restore mobility and function. Advances in new technologies, materials, techniques and pharmacological agents are particularly welcome. The journal welcomes original research, clinical studies, reviews \& evaluations, expert opinion and commentary, case reports and extended reports. The manuscript management system is completely online and includes a very quick and fair peer-review system, which is all easy to use. Visit http://www.dovepress. com/testimonials.php to read real quotes from published authors. 